\title{
Perspectivas actuales y futuras del sistema de Seguridad Social en España
}

\author{
José Antonio Panizo Robles \\ Subdirector General de Ordenación y Asistencia Jurídica del Instituto Nacional \\ de la Seguridad Social
}

\section{Introducción}

Quisiera, en primer lugar, agradecer a los organizadores de estas Jornadas que, dentro del marco de conmemoración del Centenario de la Seguridad Social, se celebran bajo el título genérico de «La Seguridad Social en el umbral del siglo XXI», para que el Instituto Nacional de la Seguridad Social esté presente en las mismas.

De acuerdo con el programa previsto, me corresponde efectuar una reflexión sobre la situación actual y las perspectivas de futuro del sistema español de la Seguridad Social. Y en esta reflexión, creo que es conveniente partir de una consideración inicial sobre lo que representa este mismo sistema.

El sistema de la Seguridad Social es un mecanismo esencial para garantizar el bienestar global de la sociedad, constituyendo de esta forma un pilar básico de la cohesión social y un instrumento de progreso, de vital importancia, que es necesario preservar en el futuro. No hay que olvidar que el propio Tratado de la Unión considera, como uno de los objetivos básicos de aquélla «lograr el más alto nivel de empleo y de protección social», considerando, además, que los sistemas de Seguridad Social «constituyen una de las señas de identidad del modelo europeo de sociedad».

Ahora bien, cuando se hace referencia a los sistemas públicos de protección social y, dentro de ellos, a los de Seguridad Social, es necesario tener en cuenta una serie de premisas básicas sobre las características de esos mismo sistemas, pues ellas nos permiten explicar en buena parte la evolución seguida por los mismos que, además, condicionan y explican su devenir futuro. 
Entre estas características, cabe destacar las siguientes:

—En primer lugar, que un sistema de Seguridad Social es «tributario de su historia». Ello quiere decir que en su evolución se ve arrastrado por las opciones que en su momento se adoptaron. Y respecto al sistema de Seguridad Social español, ya a principios de la década de los años sesenta — en línea con lo que sucedía en nuestro entorno geográfico y socio-cultural- se adoptaron unas decisiones (la configuración de un sistema de protección social fundamentalmente contributivo, basado en una modalidad financiera de reparto, con una gestión pública — sin perjuicio de presenciar la gestión privada en determinadas áreas, a través de la vía de la colaboración en la gestión-, con una unificación de sus recursos, etc.), que van a condicionar la evolución de ese sistema.

-En segundo lugar que el sistema de la Seguridad Social se instituye como un conjunto de mecanismos en orden a dar respuesta a determinadas necesidades sociales. Si las demandas sociales van modificándose a lo largo del tiempo, en lógica coherencia deben adaptarse los propios sistemas de Seguridad Social, pues en caso contrario corren el peligro bien de dejar de dar las respuestas adecuadas a esas necesidades sociales o, también, de producir una deslegitimación social.

-A su vez, que los sistemas de Seguridad Social, sin perjuicio de sus finalidades y objetivos específicos - la cobertura de las necesidades sociales- se encuadran dentro de un marco económico general, que le da soporte. Por ello, no puede perderse de vista esa interrelación entre los aspectos sociales y económicos. Si bien, el marco económico no puede desconocer los ámbitos sociales y las exigencias de la cohesión social, tampoco los sistemas de Seguridad Social pueden alejarse de las exigencias económicas, so pena de correr al corto o medio plazo serios peligros de viabilidad.

- Asimismo, que en los sistemas de Seguridad Social se deben combinar adecuadamente determinados principios, como son, de una parte, los de contribución, proporcionalidad y equidad y, de otra, el de solidaridad. Un sistema de Seguridad Social que olvide la solidaridad, difícilmente podrá ser calificado de esa forma — de Seguridad Social—; será un sistema de ahorro o de seguro, pero, insisto, no podrá hablarse propiamente de un sistema de Seguridad Social.

Pero, un sistema de Seguridad Social en el que prima, de forma esencial, el principio de solidaridad, sin tener en cuenta, o sin te- 
nerlas de forma adecuada, la exigencia de contribución, proporcionalidad y equidad, corre el peligro de convertirse en un sistema asistencial, el cual, sin perjuicio de sus características intrínsecas, difiere de lo que venimos configurando como sistema de Seguridad Social.

- De igual modo, que en los sistemas de Seguridad Social, la gestión debe estar presidida por unos principios de eficiencia $y$ eficacia, no sólo en el sentido de que se gestione más y mejor, y con el menor coste posible, sino, además, que las prestaciones se reciban por quienes se encuentran en la situación protegida y reúnan los requisitos establecidos, eliminando o reduciendo en la medida de lo posible el uso indebido o fraudulento en el acceso o en el mantenimiento de la protección.

Si cualquier Administración Pública ha de luchar de forma decidida contra el fraude, esta exigencia resulta más ineludible en el ámbito social, ya que en él — como antes señalaba- está presente un mayor componente de solidaridad, que precisa que los recursos públicos que la sociedad destina a las atenciones sociales no se dediquen a otra finalidad, puesto que esa situación - la fraudulenta- es una de las mayores causas de deslegitimación de los sistemas de la Seguridad Social y, al final, un sistema de protección social que se instaura en el fraude es objeto de una fuerte modificación.

- Por último, que, en un marco de mayor interdependencia económica y, en determinadas zonas geográficas, en las que se ha producido una mayor integración incluso política, la posible evolución de los sistemas de Seguridad Social deben estar en línea con las reformas y medidas que se adoptan en ese marco internacional y, de modo primordial, en el seno de la Unión Europea.

Por ejemplo, las modificaciones llevadas a cabo en los últimos años en el sistema de Seguridad Social de España están en línea con determinadas adaptaciones que se vienen introduciendo en los sistemas de la Seguridad Social de los países que conforman la Unión Europea. También en ellos - y valgan los ejemplos de las adaptaciones que se han introducido en los sistemas de Seguridad Social de países como Francia, Italia, Alemania, Países Bajos o los que denominamos «Estados nórdicos»- se ha sentido la necesidad de introducir elementos de racionalidad a esos propios sistemas, procurando fortalecer los mecanismos de contribución y proporcionalidad, sin descuidar al tiempo los de solidaridad.

Y este hecho no debe sorprendernos, ya que, aunque en el ámbito de la Unión Europea no exista un sistema único de Seguridad 
Social, ni esos sistemas han sido objeto de una armonización clara, sí que están presentes en los mismos unas líneas de convergencia, a veces de forma explícita y otras implícita, que impulsan a la aproximación de los propios sistemas de Seguridad Social.

\section{Situación actual del sistema de Seguridad Social en España}

En los últimos años, en el sistema español de Seguridad Social se han ido adoptando todo un conjunto de medidas que, han tenido como ejes, por una parte, potenciar los aspectos de contribución y de equidad, pero, al tiempo, incrementar la solidaridad, medidas que se han enmarcado sobre dos ejes esenciales:

- el fuerte consenso sobre los rasgos básicos del modelo de protección social y

- el convencimiento de que los sistemas de Seguridad Social han de adaptarse de forma permanente a una realidad social, siempre en transformación.

1. La existencia de un amplio consenso sobre la delimitación de nuestro sistema de Seguridad Social

En la delimitación del sistema español de Seguridad Social han jugado un papel esencial los acuerdos políticos y sociales alcanzados en los últimos años, para redefinir un modelo de protección, así como las medidas a adoptar en orden a su estabilidad y consolidación. Por ello, creo que es esencial que nos detengamos un momento en los objetivos contenidos en el «Pacto de Toledo» (compromiso político de todo el Parlamento español, alcanzado en 1995), así como en el «Acuerdo Social» de 1996, suscrito entre el Gobierno y las Organizaciones sindicales más representativas.

Por consiguiente, existe en España un compromiso político y social sobre la realidad y evolución del sistema de Seguridad Social, que puede resumirse en dos rechazos y en una afirmación, del modo siguiente:

- Hay un rechazo a la transformación radical del sistema español de Seguridad Social, basado en el reparto, por otro asentado en la capitalización, tanto por razones técnico-económicas, como por otras de solidaridad y de justicia social.

- También existe una unanimidad en rechazar que el sistema público de Seguridad Social se reduzca a un sistema de protección mí- 
nima, dejando las pensiones proporcionales y contributivas totalmente a la gestión privada.

- Por el contrario, se participa de forma unánime en la idea de que ha de continuarse con el sistema de Seguridad Social que ha ido evolucionando a lo largo del siglo XX, introduciendo las medidas que le den soporte financiero y que permitan su estabilidad y su viabilidad futuras.

Y bajo estas premisas básicas, se ha apostado por un modelo que, como es bien sabido, se articula en las siguientes modalidades de protección:

- Una modalidad contributiva de prestaciones económicas, en favor de quienes realizan una actividad laboral o profesional, mediante la cual se reconozcan prestaciones sustitutivas de los ingresos o de las rentas obtenidas a lo largo de la vida activa, y cuyo importe esté directamente relacionado con el tiempo y la cuantía de las contribuciones realizadas.

Esta modalidad se financia a través de las cotizaciones sociales abonadas por los interesados y, en su caso, por las empresas para las que prestan servicios.

- Una modalidad no contributiva de prestaciones económicas, en favor de las personas que, ante la contingencia protegida, carezcan de recursos suficientes para atender a sus necesidades, y con la finalidad de compensar esa ausencia de ingresos. La cuantía de las prestaciones es de importe fijo, si bien existen cuantías diferenciadas cuando se dan situaciones distintas de necesidad.

La financiación de las prestaciones no contributivas se efectúa por aportaciones del Estado a la Seguridad Social, de conformidad con el principio de solidaridad general que aquéllas encierran.

—Una modalidad de prestaciones de acceso universal (básicamente la asistencia sanitaria y los servicios sociales), dirigidas a toda la población y financiadas por aportaciones del Estado a la Seguridad Social.

-Por último, una modalidad de prestaciones complementarias, no sustitutivas ni alternativas a las prestaciones públicas, con la finalidad, precisamente, de complementar aquéllas.

La financiación de esta modalidad de prestaciones se efectúa a través de aportaciones de los propios interesados (o, en algunas modalidades, de sus empresas), y su gestión corresponde en su integridad al sector privado. 


\section{La necesidad de adaptación de los sistemas de Seguridad Social}

Pero aunque exista un consenso sobre la realidad de nuestro modelo de Seguridad Social, así como una defensa de sus premisas esenciales, ello no puede estar reñido sino que, al contrario, es consustancial con la constante necesidad de ir introduciendo las medidas adecuadas para que el sistema de Seguridad Social pueda seguir cumpliendo su papel y hacer frente a los retos y desafíos que se le presentan constantemente. Como ha señalado la Comisión Europea «lo que verdaderamente pondría en peligro el modelo social europeo es que no pudiera hacer frente a los problemas existentes».

En este ámbito, la Comisión de la Unión Europea ha presentado, en los últimos cuatro años, tres importantes iniciativas centradas, la primera de ellas, sobre «El futuro de la protección social», y las otras dos relacionada con la «Modernización y mejora de la protección social en Europa, en un marco de estrategia concertada», en las que se incluyen los ejes que, a su juicio, deben seguir los sistemas de la Seguridad Social para poder hacer frente al conjunto de retos y desafíos que, en los umbrales del siglo Xx, se le presentan a aquéllos y, de este modo, poder seguir cumpliendo, en la próxima centuria, su papel básico de instrumento de cohesión social y de «señas de identidad de la sociedad europea».

Para que los sistemas de protección social puedan hacer frente a esos retos y desafíos, las instancias comunitarias recomiendan, entre otras, las siguientes medidas:

a) La necesidad de consolidación de los sistemas de Seguridad Social y, más en general, de las finanzas públicas a fin de evitar el crecimiento del déficit y de gravosos servicios de deuda en el futuro, puesto que todo ello iría en detrimento del gesto en protección social, lo cual no debe estar en contradicción con un alto grado de protección social, ni puede conducir al desmantelamiento de la solidaridad, sino más bien como un reforzamiento de la solidaridad entre diferentes generaciones, en la que se basa el desenvolvimiento de los sistemas europeos de Seguridad Social.

b) La necesidad de lograr una mayor vertebración social, haciendo de los sistemas de protección social un vehículo de promoción de integración económica y social.

c) La conveniencia de adaptación o adecuación de los sistemas de protección a la naturaleza cambiante del trabajo.

d) El desafío que presenta el envejecimiento de la población europea a los sistemas de protección social y, dentro de ellos, a los sistemas de pensiones. 
e) Y, por último, la necesidad de combinar adecuadamente los mecanismos complementarios de la protección social con los de naturaleza pública.

Pues bien, si analizamos el conjunto de los retos y desafíos con los que, a juicio de la Comisión Europea, se enfrentan los sistemas de Seguridad Social, veremos que son, en grandes líneas, los mismos que se plasmaron en el Pacto de Toledo y en el Acuerdo Social de 1996.

\section{Las medidas adoptadas en la adaptación de los sistemas de protección social}

A la solución de tales problemas se han dirigido los Acuerdos parlamentario y social a que antes he hecho referencia. En los mismos se ha reflejado la voluntad de asegurar la pervivencia del modelo protector, a través de la puesta en marcha de toda una serie de medidas en el área de la protección, la financiación o la gestión, que permitan la consolidación y la racionalización del sistema, dentro de un entorno de crecimiento.

Quisiera a continuación comentar, de forma breve, alguna de las medidas adoptadas en los últimos tiempos en el ámbito de la Seguridad Social, que han tenido como finalidad -y permítanme que sea insistente en ello- la consolidación y la racionalización de nuestro sistema protector.

\subsection{La reforma financiera de la Seguridad Social}

Una de las primeras medidas adoptadas ha consistido en la culminación del proceso de separación de las fuentes financieras del sistema, adecuando las mismas a la naturaleza de las prestaciones, de forma que las de naturaleza contributiva, cuya finalidad es otorgar rentas de sustitución, encuentren su financiación en las cotizaciones sociales, mientras que las de naturaleza no contributiva se financien con aportaciones del Estado, procedentes de la imposición general, en base a la solidaridad universal que aquéllas encierran.

Esta reforma financiera, llevada a la práctica con una dosis de prudencia y de forma paulatina, ha culminado prácticamente en el año 2000 , en el que las prestaciones no contributivas ya se financien con aportaciones del Estado.

Pero, al mismo tiempo, la evolución favorable de la economía, combinada con la separación de las fuentes de financiación, ha permitido también la constitución de un fondo de reserva (con una dotación inicial de 100.000 millones de pesetas), fondo que está llamado a de- 
sempeñar un papel relevante en un modelo de Seguridad Social como el español, basado en el reparto, régimen financiero que tiene indudables ventajas para la configuración de un sistema integrado, pero que, al tiempo, requiere de mecanismos que garanticen en todo momento su equilibrio, a fin de que no se vea afectado por los altibajos de la coyuntura económica.

En línea con la reforma financiera apuntada, se ha procedido a una equiparación de las bases de cotización con los salarios reales de los trabajadores, a través del establecimiento de un único tope de cotización, aplicable a todos los grupos de cotización y categorías de trabajadores, en un proceso gradual a fin de evitar que pueda incidir de forma negativa en la competitividad y el empleo. Este proceso, que deberá culminar en el año 2002, originará un reparto más equitativo de la distribución de la carga entre sectores y en un incremento de los niveles de protección.

\subsection{La acentuación de los principios de contribución y solidaridad}

Al tiempo que se ha puesto en marcha la reforma financiera de la Seguridad Social, se ha incidido en el otro objetivo que persigue nuestro sistema de protección, consistente en reafirmar los principios que debe regir su ámbito de protección, es decir, lograr un adecuado efecto sustitutorio de rentas y propiciar la solidaridad con los sectores más desfavorecidos.

Así, en relación con la jubilación, y con objeto de incrementar la contribución y la equidad, se ha procedido a la ampliación progresiva del penado a computar para el cálculo de la base reguladora, de modo que se alcance una mayor relación entre la prestación lograda y la contribución realizada; a su vez, se ha modificado la forma de cómputo de los años trabajados a efectos de la determinación del porcentaje aplicable para determinar la cuantía de la pensión, al tiempo que se ha minorado el coeficiente reductor de la pensión de jubilación en los supuestos de personas que tienen que jubilarse anticipadamente por causas no imputables a ellos.

Dentro de la intensificación de los mecanismos de solidaridad, se ha incorporado una serie de medidas en relación con la orfandad que mejoran notablemente su ámbito de cobertura, a través de la ampliación de los límites de edad para poder ser beneficiarios de la prestación.

La misma finalidad solidaria tiene igualmente la elevación de las cuantías mínimas garantizadas a los pensionistas de viudedad menores de 60 años y con cargas familiares, así como la posibilidad de 
que se puedan causar prestaciones de muerte y supervivencia, aún cuando el trabajador fallecido no se encontrase, en el momento de su fallecimiento, en una situación de alta o de asimilación al alta.

Por último, y dentro de este ámbito de protección, se ha incorporado la garantía expresa en el mantenimiento del poder adquisitivo de las pensiones, a través de fórmulas de revalorización automática en función de la evolución real del IPC, garantía que queda reforzada con la inclusión de cláusulas de revisión y que extienden sus efectos a la totalidad de los pensionistas de la Seguridad Social, con independencia del momento o de la legislación conforme a la cual se causaron sus pensiones.

\subsection{La adecuación del sistema de protección social} a las nuevas realidades del mercado de trabajo

Otro de los retos a los que se enfrentan los sistemas de la Seguridad Social es la necesidad de adaptar los mecanismos protectores a la realidad cambiante del trabajo y de las nuevas modalidades de contratación.

La aparición de los contratos a tiempo parcial, los contratos temporales y, en definitiva, lo que ha pasado a denominarse como «trabajo atípico», ha originado la necesidad de adaptar los sistemas de Seguridad Social a estas nuevas formas de realización del trabajo.

En este ámbito, desde las instancias comunitarias se vienen proponiendo medidas que permitan que los sistemas fiscales y de cotizaciones sociales sean más favorables al empleo, en orden a favorecer a las personas en demanda de inserción laboral o que buscan su vuelta al trabajo. Con este objetivo, se ha propuesto una reducción de cotizaciones sociales, no de carácter general, sino selectiva, haciendo especial énfasis en determinados grupos de desempleados que tienen una mayor dificultad para lograr un empleo, pero sin que la reducción de las cotizaciones implique una merma en la protección social de los interesados.

Los Acuerdos de los Consejos Europeos de Essen, Cannes, Corfú, o los posteriores adoptados en las cumbres de Luxemburgo o Colonia son constantes y reiterativos en tales objetivos.

En el sistema español de protección social se ha establecido todo un conjunto de medidas dirigidas a esa misma finalidad. Tanto el Acuerdo sobre contratación y para la estabilidad del empleo de 1997, incorporados al ordenamiento jurídico a través de las Leyes 63/1997 y 64/1997, como el Real Decreto-Ley 15/1998, sobre trabajo a tiempo parcial o los correspondientes Planes de Fomento del Empleo para los ejercicios 1999 y 2000, han tenido unos efectos positivos, ya que: 
a) De una parte, se ha mejorado la protección efectiva de los trabajadores contratados en esta modalidad de contratos. De este modo, los trabajadores, contratados en contratos para la formación o en contratos a tiempo parcial, han pasado a tener una protección social completa y en términos similares a los trabajadores que prestan servicios en contratos a jornada completa, suprimiéndose las lagunas de protección existentes con anterioridad, las cuales - en especial, en los contratos a tiempo parcial de jornadas más reducidas- podían ser significativas.

Incluso, en lo que se refiere a los trabajadores a tiempo parcial, se han establecido mecanismos de "discriminación positiva», que les permiten acceder a la protección social de forma efectiva, sobre todo respecto de aquellas prestaciones económicas en cuyo acceso se exigen amplios períodos de cotización.

b) La bonificación de las cotizaciones sociales se ha dirigido básicamente a la contratación estable. No se trata sólo de lograr que el mercado de trabajo ofrezca a los trabajadores un empleo, sino también que ese empleo sea de calidad, como modo de ir terminando con la fuerte tasa de temporalidad de que adolecía el empleo en España, con los beneficios consiguientes para los trabajadores, en cuanto a la seguridad y la estabilidad en el empleo, pero también a las empresas, puesto que la mayor seguridad y estabilidad laboral favorece la productividad.

c) Una apuesta decidida por la formación, como medio de incrementar el grado de «empleabilidad» de los trabajadores. Debido a una multiplicidad de causas (entre las que se encuentran los avances tecnológicos) la situación del mercado de trabajo se ha modificado sustancialmente en los últimos años.

Con anterioridad, como norma general, un trabajador adquiría unas aptitudes concretas que le permitían el desarrollo de unos determinados cometidos laborales; ante la pérdida de un empleo, era factible que esos conocimientos y aptitudes adquiridos le posibilitasen, con mayor o menor rapidez, encontrar un nuevo trabajo adaptado a aquéllos.

Sin embargo, los fuertes avances tecnológicos y el surgimiento de la «sociedad del conocimiento» están modificando sustancialmente la situación anterior. Se requieren de unos conocimientos concretos, en permanente cambio, que exigen una constante formación y la puesta al día de los conocimientos previamente adquiridos, cuando no la adquisición de nuevos conocimientos para volver a insertarse en el mercado de trabajo, cuando de forma temporal se ha salido de aquél. Por ello, el in- 
cremento del «grado de empleabilidad» es una exigencia necesaria en la lucha contra el desempleo, como se viene recomendando desde las instancias comunitarias y, más recientemente, desde la Cumbre de Jefes de Estado y de Gobierno de la Unión Europea, celebrada en Lisboa.

Con tal finalidad, en los últimos Planes de Empleo se ha apostado fuertemente por un incremento de la formación como medio para reducir las todavía fuertes tasas de paro que tiene la sociedad española, las cuales, si bien se han reducido espectacularmente en la pasada legislatura, siguen siendo un desafío básico de nuestra sociedad.

d) Por último, la puesta en marcha de programas que combinan la percepción de una ayuda de desempleo, con programas de formación, entre los que se encuentran la «renta activa de inserción», a través de la cual un trabajador desempleado suscribe un compromiso con las Instituciones Públicas de realizar cursos de formación, que le posibilite para su inserción laboral, percibiendo, mientras efectúa estos cursos, una prestación social.

\subsection{La adaptación de la protección social a la evolución}

de la participación de hombres y mujeres en la vida profesional

La participación de la mujer en el mercado de trabajo (que supone una de las grandes transformaciones sociales de la segunda mitad del siglo XX) continuará creciendo en los próximos años, lo que requiere encontrar nuevos mecanismos que permitan conciliar la vida profesional y familiar, así como, en la medida en que sea posible, la aplicación de forma progresiva de la individualización de los derechos.

En España, hace unos meses se ha aprobado una Ley que tiene como finalidad la promoción de la conciliación de la vida familiar y laboral de las personas trabajadoras, en un marco de esa mayor participación de la mujer en el trabajo, así como de igualdad de oportunidades hombre/mujer.

Dentro de este objetivo, y en el marco de determinadas Directivas comunitarias:

- se han ampliado los períodos de suspensión del contrato de trabajo (y de la correspondiente prestación económica de la Seguridad Social) por maternidad y adopción, mejorando, al tiempo, la propia prestación social;

- se ha establecido una nueva prestación de Seguridad Social, para cubrir la eventualidad de que el desarrollo del puesto de trabajo presente riesgo para la trabajadora embarazada o para el feto, 
cuando aquélla no pueda ser trasladada a un puesto de trabajo compatible con su estado;

— se han mejorado los sistemas de reducción de la jornada, permisos o de excedencia (con reserva de puesto de trabajo), en los casos en que un trabajador/trabajadora haya de dedicarse a los cuidados de un hijo menor o de otro familiar, que no pueda valerse por sí mismo, a causa de la edad, de la enfermedad o de la incapacidad;

- se han establecido determinadas garantías en el mantenimiento del puesto de trabajo, declarándose nula la extinción de la relación laboral de una persona que tenga suspendido su contrato de trabajo, a causa de las situaciones de riesgo durante el embarazo, maternidad, adopción o cuidado de un hijo menor o de otro familiar;

-o, por último, se han eliminado la totalidad de las cotizaciones sociales a cargo de las empresas, cuando las mismas contraten a un trabajador desempleado, para sustituir a un trabajador/trabajadora que tenga suspendido su contrato de trabajo ante las situaciones que les acabo de enumerar (es decir, riesgo durante el embarazo, maternidad, adopción o cuidado de un familiar).

\section{Perspectivas de futuro en la evolución del sistema de Seguridad Social de España}

Como les indicaba al principio, los sistemas de Seguridad Social se encuentran sometidos a una constante y continua transformación, a fin de que puedan ir dando respuesta a las necesidades cambiantes de la protección. Por ello, resulta necesario, de una parte, proceder a la corrección de los desequilibrios que puedan presentarse y, de otra, adecuar sus mecanismos para hacer frente de manera eficaz a los retos que habrán de asumir al corto y medio plazo, y que suponen las claves en que tendrá que desarrollarse la Seguridad Social en el siglo XXI.

\section{El reto demográfico}

Dentro de tales retos destaca, por su importancia, la evolución demográfica que se caracteriza por un paulatino crecimiento del número de personas mayores, originado tanto por el descenso de la natalidad, como también por los avances producidos en los ámbitos de la medicina y de la salud pública, con su repercusión en el incremento de la esperanza de la vida.

España no es una excepción en el conjunto de los países occidentales respecto del envejecimiento de la población, si bien en nuestro caso 
este fenómeno se está produciendo con un cierto retraso, en relación con los países europeos, debido a una peculiar evolución demográfica en el siglo XX, caracterizada por una importante caída de los nacimientos en la década siguiente a la Guerra Civil y por un notable aumento durante la década de los 60 y principio de los 70 .

No obstante, las cifras muestran ya una clara tendencia hacia el envejecimiento de la población. La población española mayor de 65 años, que representaba un $11,2 \%$ a principio de los 80 , será, a comienzos del siglo próximo, cerca del 17\%; representará más del $18 \%$ en el 2010 y alcanzará el 20,6\% en el 2020.

Estos cambios demográficos seguramente provocarán el nacimiento de una sociedad distinta, en la que parte de los valores actuales dejarán de tener presencia o, al menos, con la intensidad actual. De igual modo, esa nueva sociedad llevará consigo un aumento de las demandas y necesidades de las personas mayores, a las que habrá que responder de forma adecuada, favoreciendo su integración social, económica y personal, desde un ámbito que garantice y eleve su calidad de vida.

\section{Las transformaciones sociales}

El reto demográfico, con ser básico, no es el único al que deberá atender la Seguridad Social, puesto que deberá prestar atención a las transformaciones familiares, a la aparición de nuevas formas de marginación social o al mantenimiento de un entorno que garantice el crecimiento económico y la creación de empleo.

Por lo que respecta a las estructuras familiares, se están produciendo profundos y continuos cambios que son consecuencia tanto de la incorporación de la mujer al mercado de trabajo y del surgimiento de nuevas formas de convivencia, como también del propio cambio de los hogares españoles caracterizados por una más tardía emancipación de los hijos y, como consecuencia de ello, por un mayor período de dependencia de los padres.

Se hará necesario, por tanto, redimensionar las políticas de protección social dirigidas al entorno familiar y diversificar las prestaciones en base a las cambiantes circunstancias a las que nos enfrentamos, con especial incidencia en la satisfacción de las necesidades de los colectivos más débiles.

A su vez, resulta preciso tener en cuenta el posible incremento de diferentes formas de marginación social, que surgen como efecto del desarrollo económico o las nuevas formas de producción, orientadas en parámetros de eficacia y capacitación, las cuales pueden marginar a todo un conjunto de personas que por su edad, discapacidad o perte- 
nencia a determinados grupos no pueden competir en plan de igualdad y se ven excluidas del mercado de trabajo.

Se corre el peligro del agravamiento de un fenómeno de dualización social, el cual puede desembocar en situaciones de pobreza, que será necesario atajar con medidas que propicien una mayor integración sociolaboral de los colectivos afectados y/o con prestaciones que permitan satisfacer sus necesidades básicas, en orden a evitar una importante fractura social de importantes consecuencias.

Para evitar este problema, desde las instancias comunitarias se propone mejorar la capacitación de las personas, promover un acceso más amplio al conocimiento y a las oportunidades de integración social.

Asimismo, resulta imprescindible que la Seguridad Social se incardine en un marco de crecimiento y creación de riqueza y de generación de empleo. Esta prioridad, en un entorno cada vez más abierto y globalizado, adquiere día a día mayor importancia y exigirá de mecanismos de financiación del modelo protector, de modo que, procurando la suficiencia de la cobertura social, sea posible neutralizar cualquier desviación en el destino final de los recursos asignados.

No hay que olvidar que la lucha contra el desempleo y la inserción en el mercado de trabajo es la mejor salvaguardia contra la exclusión social.

En este marco y como ha recordado la Cumbre de Lisboa, de marzo del presente año, los sistemas de protección social han de adaptarse como parte del bienestar activo, en orden a garantizar la buena salud del mercado laboral y su propia sostenibilidad a largo plazo, con una población en proceso de envejecimiento, que permita la promoción de la inclusión social y la igualdad de los sexos.

\section{Perspectivas de futuro en la evolución del sistema de la Seguridad Social}

Los retos y desafíos que se ciernen sobre los sistemas de protección social va a requerir, sin duda, de todo un conjunto de medidas que dé solución a las demandas sociales y que, al tiempo, se sitúe en un contexto que posibilite mantener y asegurar los actuales niveles de protección social dentro de un entorno económico-financiero equilibrado y estable, medidas entre las que se encuentran:

3.1. Aprovechar las potencialidades y las experiencias de los trabajadores de más edad

Los sistemas de pensiones, en buena parte de nuestros países, están configurados de tal forma que una persona, al llegar a una determinada 
edad, ha de abandonar totalmente su actividad, pasando a percibir la correspondiente pensión de jubilación o de vejez. Incluso, hace algunas décadas, se proponía adelantar la edad de acceso a la jubilación, de modo que se permitiese acceder al mercado de trabajo a las generaciones más jóvenes.

La experiencia acumulada ha puesto de manifiesto la falsedad de esa especie de "conflicto» entre generaciones, y que la promoción del adelantamiento en el acceso a la jubilación no ha permitido la inserción en el mercado de trabajo de trabajadores jóvenes. Además, las fórmulas actuales de jubilación pueden estar implicando un «derroche social», puesto que se desperdicia la experiencia adquirida por esos trabajadores, así como la posibilidad de que puedan seguir desempeñando un papel activo en la sociedad.

En este contexto, entiendo que la propuesta ha de consistir en incentivar la prolongación en la vida activa de tales trabajadores, con las indudables ventajas que tal hecho supone para la viabilidad de los sistemas de pensiones (en un contexto de progresivo envejecimiento de la población), así como para la autonomía y autoestima de los propios trabajadores.

3.2. La continuación en la potenciación de los principios de contribución y de equidad

Uno de los ejes de la reforma de 1997 consistió en la potenciación de los principios de contribución y de equidad, dentro de los ámbitos contributivos de la protección. Pues bien dichos objetivos han de proseguir en el futuro, de modo que, por una parte, se guarde la mayor correspondencia entre la cotización y prestación y, por otra, de que a igual esfuerzo de contribución se alcance un nivel de protección similar.

\subsection{La creación de empleo}

Una de las garantías de la pervivencia y mejora del sistema de Seguridad Social se encuentra en la generación de empleo. Las políticas adoptadas en la pasada legislatura — basadas en la articulación de figuras contractuales más adaptadas a la realidad de nuestro mercado laboral, así como en el establecimiento de bonificaciones sociales que, al tiempo que no ponían en cuestión el equilibrio financiero del sistema apostaban claramente por el empleo estable y de calidad - han logrado una dinamización del mercado laboral, como queda de manifiesto en el aumento de la afiliación que, desde marzo de 1996, ha crecido en más de 2,5 millones de personas. 
En estos días, se ha hecho público que, a finales del mes de mayo y por primera vez en nuestra historia de Seguridad Social, el número de afiliados superó la barrera de los 15 millones (para situarse, exactamente, en 15.049.683 personas); de igual modo, el Régimen General - que agrupa cerca del 70\% del total de afiliados- también superó la cifra de afiliados de 11 millones (se situó en 11.085.040 afiliados).

Este dato supone una garantía adicional para el sistema de Seguridad Social, ya que afecta a otro de los factores esenciales para asegurar el equilibrio de la Seguridad Social, cual es el mantenimiento o mejora de la relación entre el número de afiliados y el de pensionistas. Esta relación que se situaba en apenas un 2\% en 1996 ha subido hasta el 2,25.

\subsection{La mejora de la protección social}

Dentro del mantenimiento de la protección social, en un marco de crecimiento económico sostenido, resulta necesario un incremento de los mecanismos solidarios, que aumenten la protección y la lucha contra la dualización social. Sería socialmente inaceptable que una sociedad que mejora económicamente no atienda de manera adecuada las necesidades de cobertura de sus ciudadanos, con especial intensidad en los colectivos más desfavorecidos.

En esta línea, la mejora de las pensiones de cuantía más reducida y la regulación más favorable para estos colectivos de la incompatibilidad de las pensiones (por ejemplo, en las pensiones de viudedad o en el SOVI) resultan convenientes para procurar esa mejora de la protección.

\subsection{La consideración del principio de paridad y la conversión de los derechos derivados en derechos individuales}

La propia evolución de la sociedad, con la incorporación de la mujer al mundo del trabajo, la mayor corresponsabilidad de hombres y mujeres, y la aplicación, cada vez con mayor intensidad, del principio de paridad, también debe tener su efecto en la evolución de los sistemas de Seguridad Social.

Aún hoy, la posición de hombres/mujeres en la sociedad no son equiparables, ni se ha alcanzo en su plenitud la aplicación del principio de igualdad de oportunidades. El propio anuncio de la Comisión Europea en orden a impulsar una nueva Directiva para favorecer la discriminación positiva de la mujer pone de relieve que el principio de paridad todavía no se ha logrado, lo cual tiene también su reflejo en los sistemas de protección social, ya que, dada su estructura, no son, en sí mismos, la solución de los problemas de la desigualdad; antes 
bien las instituciones de Seguridad Social han partido, con frecuencia de la existencia de esa desigualdad, estableciendo mecanismos que la compensasen.

Pero, al tiempo que la igualdad se va haciendo más efectiva, surge la problemática de los derechos derivados (tan frecuentes en los momentos de implantación de los sistemas de protección social), así como la necesidad de que, mediante una etapa larga de progresividad, los mismos vayan convirtiéndose en derechos propios, en cuya configuración habrán de tenerse especial consideración los períodos que, en sustitución de un trabajo lucrativo, los miembros de una comunidad dediquen para el cuidado de menores o de personas de edad o enfermas, en línea con el camino emprendido en la Ley de conciliación de la vida familiar y laboral de las personas trabajadoras.

\subsection{El perfeccionamiento de los sistemas complementarios}

Las transformaciones sociales, en particular el proceso de envejecimiento, ha suscitado un debate vivo sobre la viabilidad de los actuales sistemas públicos de pensiones y sobre las alternativas para dotarlos de mayor estabilidad.

Aunque existen dos modelos básicos de financiación de las prestaciones económicas (el de reparto y el del ahorro individual), el debate y el análisis sobre las diferentes modalidades de financiación han permitido alcanzar, al menos en el ámbito europeo, algunas conclusiones como:

- Tanto en los sistemas de reparto como en los de capitalización, hay un hecho insoslayable cual es que el PIB ha de repartirse entre personas cuyos ingresos proceden directamente de su participación en la vida económica y las que no cuentan con tales recursos.

- Cuando unos sistemas han alcanzado una importante madurez, una reforma radical de los sistemas públicos de pensiones no se presenta como posible, dados los enormes costes que ello supone.

En esta materia, se hace preciso efectuar un contexto más seguro para los regímenes complementarios, que, sin alterar los elementos del sistema de reparto, permitan a los pensionistas el mantenimiento o la mejora de su nivel de vida después de la jubilación. En España, en este ámbito se han llevado a cabo determinadas medidas que propician y posibilitan un mayor desarrollo de los mecanismos complementarios de la Seguridad Social, en el marco de una fiscalidad más favorable a los mismos, pero siempre, e insisto en ello, con un carácter complementa- 
rio y nunca sustitutivo de los regímenes públicos, obligatorios y basados en el sistema de reparto.

Esa es, al menos, la intención que se desprende de las últimas comparecencias de los responsables en la materia, en las que se ha abogado por el impulso de los planes de pensiones, la suscripción de planes individuales por mujeres que carezcan de rentas de trabajo o la conveniencia de que la previsión complementaria se incluya en el marco de la negociación colectiva.

Creo que ya he consumido el tiempo disponible y no quisiera cansarles más. Solamente una última reflexión: los retos y desafíos sobre los sistemas de Seguridad Social son importantes, por lo que son precisas medidas, que, al igual que sucedió en los años 1995 y 1996, cuenten con los mayores respaldos sociales y políticos.

La apertura de las mesas de diálogo social entre el Gobierno y los Agentes sindicales, de una parte, y entre la globalidad de los agentes sociales, por otra, así como la constitución en esta legislatura de la Comisión parlamentaria no permanente sobre el «Pacto de Toledo», son un buen precedente para que los compromisos socio-políticos sobre el modelo de la Seguridad Social permanezcan vigentes entre nosotros y que las medidas que se adopten cuenten con el mayor respaldo social.

Unicamente, volver a agradecer a los Organizadores por su invitación a estar presente en estas Jornadas conmemorativas del Centenario de la Seguridad Social y a todos ustedes por su amable atención. 